\title{
Hydration dependence of myoglobin dynamics studied with elastic neutron scattering, differential scanning calorimetry and broadband dielectric spectroscopy
}

\author{
Margarita Fomina, Giorgio Schirò ${ }^{1}$, Antonio Cupane * \\ Department of Physics and Chemistry, University of Palermo, via Archirafi 36, 90123 Palermo, Italy
}

\section{H I G H L I G H T S}

- Myoglobin dynamics is studied as a function of hydration (h).

- Mb/hydration water relaxations speed up and glass transition temperatures decrease as $\mathrm{h}$ increases.

- Amplitudes of the protein equilibrium fluctuations increase with $\mathrm{h}$.

- The onset temperatures of the Protein Dynamical Transition are hydration independent.

- Connection between protein PDT and hydration water liquid-liquid transition is suggested.
G R A P H I C A L A B S T R A C T

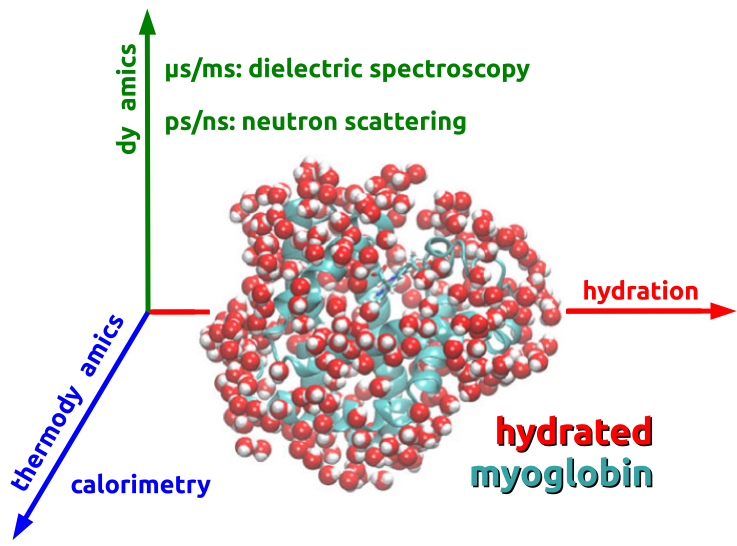

\begin{abstract}
A B S T R A C T
In this work we present a thorough investigation of the hydration dependence of myoglobin dynamics. The study is performed on $\mathrm{D}_{2} \mathrm{O}$-hydrated protein powders in the hydration range $0<\mathrm{h}<0.5\left(\mathrm{~h} \equiv \operatorname{gr}\left[\mathrm{D}_{2} \mathrm{O}\right] / \mathrm{gr}[\right.$ protein] $)$ and in the temperature range 20-300 K. The protein equilibrium fluctuations are investigated with Elastic Neutron Scattering using the spectrometer IN13 at ILL (Grenoble), while the relaxations of the protein + hydration water system are investigated with Broadband Dielectric Spectroscopy; finally, Differential Scanning Calorimetry is used to obtain a thermodynamic description of the system. The effect of increasing hydration is to speed up the relaxations of the myoglobin + hydration water system and, thermodynamically, to decrease the glass transition temperature; these effects tend to saturate at $h$ values greater than $\sim 0.3$. Moreover, the calorimetric scans put in evidence the occurrence of an endothermic peak whose onset temperature is located at $~ 230 \mathrm{~K}$ independent of hydration. From the point of view of the protein equilibrium fluctuations, while the amplitude of anharmonic mean square displacements is found to increase with hydration, their onset temperature (i.e. the onset temperature of the well known "protein dynamical transition") is hydration independent. On the basis of the above results, the relevance of protein + hydration water relaxations and of the thermodynamic state of hydration water to the onset of the protein dynamical transition is discussed.
\end{abstract}

C 2013 Elsevier B.V. All rights reserved.

\footnotetext{
* Corresponding author.

E-mail address: antonio.cupane@unipa.it (A. Cupane).

${ }^{1}$ Present affiliation: CNRS - Institut de Biologie Structurale, 41 rue Jules Horowitz, 38027 Grenoble, France.
} 


\section{Introduction}

Proteins are characterized by a large structural heterogeneity. They assume an overall 3-dimensional arrangement corresponding to the biologically active conformation; however, owing to the high flexibility and to the non-covalent interactions stabilizing their structure, they can explore, by thermal motions, a large number of conformational substates [1]. As a consequence, protein dynamics ranges over a large time-space domain, from local harmonic vibrations (femtoseconds), to collective vibrations (picoseconds), to molecular groups rotations (picoseconds) to local molecular rearrangements (pico/nanoseconds) up to relative movements of larger groups of the protein (micro-milliseconds).

The exploration by proteins of conformational substates is dynamically reflected in the activation of anharmonic atomic fluctuations; this anharmonic activation is deemed essential for protein functioning. The main onset of this kind of motions in hydrated proteins is the well-known "protein dynamical transition" (PDT), a steep increase in the amplitude of atomic motions revealed at about $220 \mathrm{~K}$ by different techniques like, e.g., elastic neutron scattering (ENS) [2]. The physical origin of this transition is still unclear, and different models have been proposed to explain it [3-11]. There is however a general consensus about the essential role played by solvent (although not necessarily water [12]) in this phenomenon. In fact, for dry protein powders, no PDT is observed. It is therefore well accepted that protein dynamics is intimately coupled to the properties of the surrounding medium which, in the biological environment, is mainly water.

Studies on the hydration dependence of protein dynamics are therefore important, both from the point of view of Biophysics/ Soft Matter (hydrated proteins may help understanding the behavior of complex systems like e. g. glasses) and from a more biological point of view since hydration/dehydration may be relevant in the regulation of protein function and in cryopreservation. This notwithstanding, systematic studies on the hydration dependence of protein dynamics are lacking. Among the few works present in the literature, it is relevant to mention those by Khodadadi et al., 2008 [13] (on hydrated lysozyme powders) and by Jansson et al., 2011 [14] (on myoglobin hydrated powders, in which however water/glycerol mixtures were used as "solvent").

In this work we present a systematic study of the hydration dependence of $\mathrm{D}_{2} \mathrm{O}$-hydrated myoglobin powders, in the hydration range $0 \div 0.5$ gr $\mathrm{D}_{2} \mathrm{O} / \mathrm{gr}$ protein. In view of the wide time scale involved in protein dynamics, we utilize ENS (time scale hundreds of picoseconds) and broadband dielectric spectroscopy (BDS, time scales ranging from hundreds of nanoseconds to seconds or more). Besides the different timescales, it should also be kept in mind that the two techniques probe different types of motions. In fact, ENS on $\mathrm{D}_{2} \mathrm{O}$-hydrated protein powders monitors essentially motions of the non-exchangeable hydrogen atoms, evenly distributed over the entire protein, that include e.g. methyl groups rotations and librational/translational-like motions of the amino acid side chains. On the other hand BDS, although covering a broad frequency range, measures motions of all the dipoles in the sample, so that assignment of the observed relaxations to specific groups (e.g. amino acid side chains and/or hydration water) is often very difficult. In this work we also use Differential Scanning Calorimetry (DSC), in order to obtain a thermodynamic description of the systems studied.

Aim of the work is to contribute towards a deeper understanding of the dynamic/thermodynamic properties of the protein + hydration water system. We are also convinced that studies on the hydration dependence will help clarifying highly debated issues like the physical origin of the PDT and its relation with the glass transition of the system and with the protein/hydration water dielectric relaxations.

\section{Materials and methods}

\subsection{Samples preparation}

Lyophilized horse heart myoglobin was purchased from SigmaAldrich (St. Louis, MO). Met-Mb hydrated powders for neutron scattering experiments were prepared with the following procedure: a solution of horse $\mathrm{Mb}$ in $\mathrm{D}_{2} \mathrm{O}$ (concentration $50 \mathrm{mg} / \mathrm{ml}$ ) was held at room temperature for approximately $24 \mathrm{~h}$, centrifuged for $20 \mathrm{~min}$ at $10{ }^{\circ} \mathrm{C}$ and subsequently re-lyophilised; the resulting powder was held for about $30 \mathrm{~h}$ under vacuum at $45{ }^{\circ} \mathrm{C}$ and considered our dry $(\mathrm{h}=0)$ sample. We are aware of the fact that the above procedure is unable to remove the water tightly bound to charged groups on the protein surface $[15,16]$ and amounting to $\sim 2 \% \mathrm{w} / \mathrm{w}$. The powder was then held in a controlled $\mathrm{N}_{2} / \mathrm{D}_{2} \mathrm{O}$ atmosphere in order to obtain the desired hydration levels ( $\mathrm{h}=$ gr $\mathrm{D}_{2} \mathrm{O} / \mathrm{gr}$ protein) that were estimated by measuring the powder mass change.

For calorimetric and dielectric spectroscopy measurements, Hydrogen/Deuterium exchange was achieved by hydration under $\mathrm{D}_{2} \mathrm{O}$ atmosphere followed by vacuum drying; this hydration-dehydration procedure was repeated several times (at least 6) so as to guarantee that most of the exchangeable $\mathrm{H}$ atoms are replaced by $\mathrm{D}$ atoms. The D-exchanged protein powder was carefully dried under vacuum at room temperature for one day and the obtained powder was considered as our dry $(h=0)$ sample. Following Jansson et al., 2011 [14], the dry powder was thoroughly mixed with the desired amounts of $\mathrm{D}_{2} \mathrm{O}$; the mixing procedure was performed by repeatedly adding small amounts of $\mathrm{D}_{2} \mathrm{O}$ to the sample until an homogeneous mixture was obtained and the final hydration level $\mathrm{h}$ was determined by measuring the mass change of mixture. To further ensure homogeneity, the samples were left to equilibrate for at least 10 days before measurements. Note that this procedure for obtaining hydrated samples is different from that previously used by Schirò et al., 2009, 2013 [17,18] for obtaining samples at $\mathrm{h}=0.3$ and $\mathrm{h}=0.5$.

\subsection{Elastic Neutron Scattering (ENS) measurements and data analysis}

ENS measurements as a function of temperature were performed on the thermal ( $\lambda=2.23 \AA$ ) high-energy resolution backscattering spectrometer IN13 (Institut Laue-Langevin, Grenoble, France) that is characterized by a very large momentum transfer range $(0.2<Q<4.9 \AA-1)$ with a good and nearly Q-independent energy resolution. IN13, therefore, allows to access the space and time windows of 1-6 $\AA$ and $0.1 \mathrm{~ns}$, respectively. In our experiments the energy resolution was fixed to $8 \mu \mathrm{eV}$. The neutron beam scattered from the sample was reflected in almost perfect backscattering geometry by $\mathrm{CaF}_{2}$ analyzers to be finally collected by $35{ }^{3} \mathrm{He}$ detectors in the region $1.1<Q<4.9 \AA^{-1}$ and by a position sensitive detector (PSD) in the low $Q$ region $\left(0.2<Q<0.8 \AA^{-1}\right)$. The elastic energy value $(\omega=0)$ was kept fixed within $3 \mu \mathrm{eV}$ of accepted tolerance.

The elastic scattering intensities $\left(I_{\mathrm{el}}(Q) \equiv S(Q, \omega=0)\right)$, suitably corrected for the empty sample holder contributions, were normalised with respect to the lowest temperature measurement to compensate for spurious background contributions and detector efficiency. No Bragg peaks due to crystalline water were observed at any of the measured temperature points, except possibly at $\mathrm{h}=0.5$ where the data at the involved Q values were discarded. In all the experiments, the sample thickness was suitably chosen to minimize the neutron absorption from the sample, thus avoiding correction from multiple scattering contributions. A transmission of about $88 \%$ was guaranteed using $1 \mathrm{~mm}$ thick Al flat sample holder.

ENS data were analyzed with a standard procedure $[19,20]$ to obtain the experimental MSD of non-exchangeable $\mathrm{H}$ atoms. The usual definition MSD $=-d \ln \left[S(\omega=0, \mathrm{Q}] / d \mathrm{Q}^{2}\right.$ was used in the $\mathrm{Q}$ range satisfying the gaussian approximation [21]. 


\subsection{Broadband Dielectric Spectroscopy (BDS) measurements and data analysis}

Dielectric measurements were performed in the frequency range $10^{-2}-10^{7} \mathrm{~Hz}$ and in the temperature range 143-293 $\mathrm{K}$ using a Novocontrol Alpha analyzer equipped with the Quatro cryogenic system. Samples were sealed in steel sample holders with Teflon spacers; inner diameter and thickness were 28 and $1 \mathrm{~mm}$, respectively. The measurements protocol was as follows: the samples were cooled to $143 \mathrm{~K}$ at a rate of about $5 \mathrm{~K} / \mathrm{min}$; after equilibration at $140 \mathrm{~K}$ the temperature was increased by steps and dielectric spectra were collected at constant temperature, every 5 or $2.5 \mathrm{~K}$; 20 min equilibration was allowed at each temperature whose stability was controlled within $0.2 \mathrm{~K}$. The stability of hydration level during the measurements was checked by weighting the samples just before and just after each experiment. In order to avoid the presence of excessive hydration dependent conductivity contributions we decided to use teflon coated ( $<100 \mu \mathrm{m}$ thickness) cells and not to analyze the relaxation peaks occurring at low frequency in high temperature spectra where spurious peaks related to teflon are expected [22]. In fact, we are aware of the debate existing in the literature about the use of blocking electrodes coated with teflon [23-25].

All dielectric spectra, namely the complex permittivity $\varepsilon^{*}$ vs frequency $\omega$, were fitted (real and imaginary part simultaneously) by a combination of Havriliak-Negami (HN) functions describing each relaxation process:

$\varepsilon=\varepsilon_{\infty}+\sum_{j} \frac{\Delta \varepsilon_{j}}{\left[1+\left(i \omega \tau_{j}\right)^{\beta_{j}}\right]^{\gamma_{j}}}$

where $\varepsilon_{\infty}$ is the high frequency limit of permittivity, $\Delta \varepsilon_{j}$ is the dielectric relaxation strength and $\tau_{j}$ is the relaxation time of the $j$-th relaxation process. The empirical parameters $0<\beta \leq 1$ and $0<\gamma \leq 1$ describe respectively the broadening and the asymmetry of each relaxation process. In order to reduce possible fitting uncertainties the variability range of parameters $\beta$ and $\gamma$ was limited to $\pm 10 \%$. The temperature dependence of relaxation times was fitted in terms of the Vogel-FulcherTamman law (VFT): $\tau=\tau_{0} \cdot \exp \left[\mathrm{DT}_{0} /\left(\mathrm{T}-\mathrm{T}_{0}\right)\right]$ or of the Arrhenius law: $\tau=\tau_{0} \cdot \exp [\Delta \mathrm{H} / \mathrm{RT}]$.

The "dielectric" glass transition temperature $\mathrm{T}_{100}$ was determined as the temperature at which the relaxation times reach the value of $100 \mathrm{~s}$.

\subsection{Differential Scanning Calorimetry (DSC) measurements and data analysis}

Calorimetric measurements were performed using a Diamond DSC Perkin-Elmer calorimeter with a Cryofill device using liquid nitrogen as a cold source. Indium was used as a standard to calibrate temperature and heat flow. Heat flow error is $0.05 \mathrm{~mW}$. Samples of Mb powders were sealed in steel pans of $\sim 60 \mu \mathrm{l}$. Samples were first cooled to $123 \mathrm{~K}$ with a cooling rate of $20 \mathrm{~K} / \mathrm{min}$; then calorimetric upscans from 123 to $303 \mathrm{~K}$ were performed with heating rate of $20 \mathrm{~K} / \mathrm{min}$. An empty sealed pan was used as a reference. The baseline was measured at the same scan rate with no pans in the furnace. The specific heat capacity of the sample was evaluated by subtracting the baseline and then dividing by the scan rate and the sample mass.

The onset temperature of glass transition, i.e. the temperature value at the onset of the leap of specific heat at low temperature, $T_{g}$ onset, and the step in the specific heat, $\Delta C_{p}$, were determined with standard methods [26].

\section{Results and discussion}

\subsection{ENS}

Total MSD values are reported in the upper panel of Fig. 1 as a function of temperature for Mb powders at different hydration levels; data
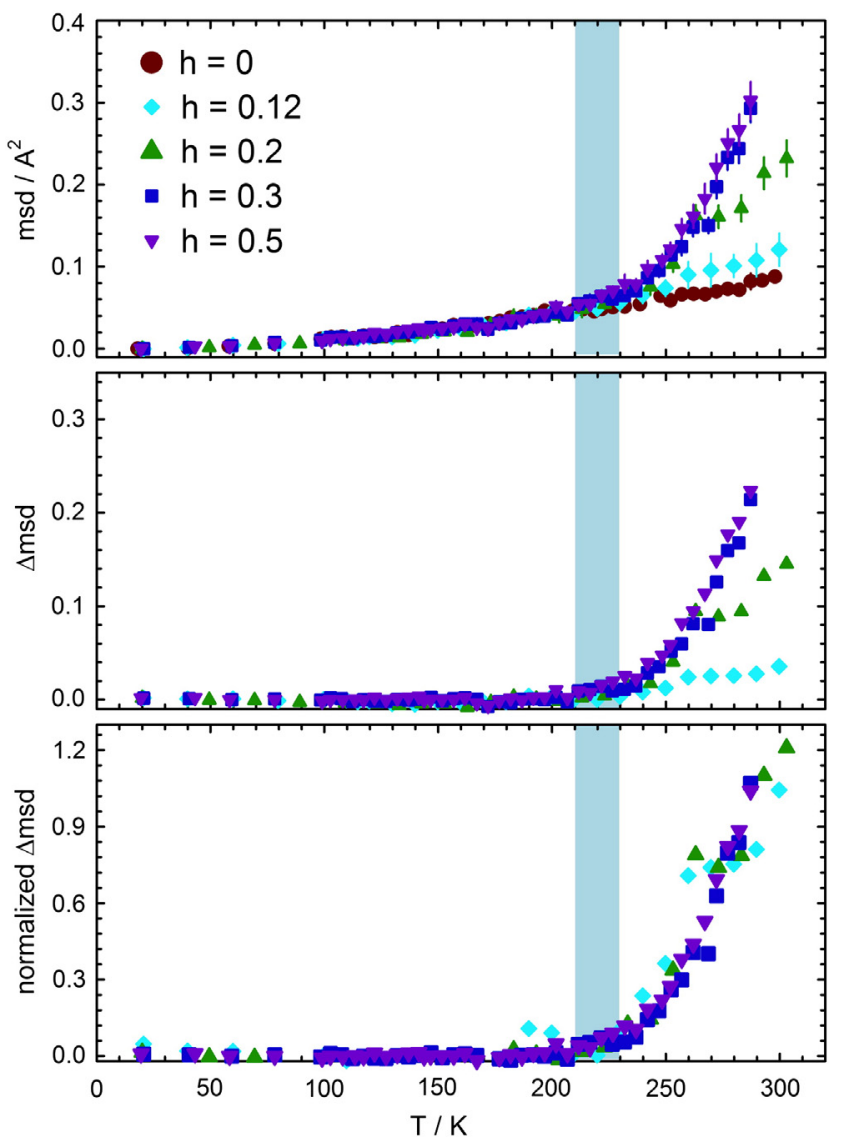

Fig. 1. MSD of non-exchangeable $\mathrm{H}$ atoms of Mb hydrated powders as a function of temperature at different hydration levels. Red circles: $\mathrm{h}=0$; cyan diamonds: $\mathrm{h}=0.12$; green triangles up: $h=0.2$; blue squares: $h=0.3$; violet triangles down: $h=0.5$. Upper, middle and bottom panels refer to total MSD, $\triangle \mathrm{MSD}=\operatorname{MSD}(\mathrm{h})-\mathrm{MSD}(\mathrm{h}=0)$, and normalized $\Delta \mathrm{MSD}=\Delta \mathrm{MSD}(\mathrm{T}) / \Delta \mathrm{MSD}(\mathrm{T}=290 \mathrm{~K})$ respectively. The blue zone highlights the temperature interval where the onset of the PDT is observed. (For interpretation of the references to color in this figure legend, the reader is referred to the web version of this article.)

relative to the "dry" powder sample are also reported. To obtain the MSD related to the PDT we adopt the procedure already used in previous publications of our group $[8,20]$, i.e. we subtract from the MSD measured at a given hydration those measured for the dry sample $(h=0)$. In this way the contributions related to harmonic motions and to methyl groups activation (almost hydration independent [27]) are subtracted while contributions related to the PDT are highlighted. $\triangle \mathrm{MSD}$ values $[\triangle \mathrm{MSD}=\operatorname{MSD}(\mathrm{h})-\mathrm{MSD}(\mathrm{h}=0)]$ are reported in the middle panel: a pronounced dependence on hydration is observed, the effect saturating at $\mathrm{h}>0.3$. To ascertain whether hydration influences the $\triangle \mathrm{MSD}$ amplitudes or their onset temperature (or both), we normalized the $\triangle \mathrm{MSD}$ to their high temperature values; normalized $\triangle \mathrm{MSD}$ are reported in the bottom panel and one clearly sees that normalized data superimpose, independent of hydration. This clearly shows that hydration affects the amplitude of anharmonic fluctuations related to the PDT (in agreement with the suggested plasticizing role of hydration water [28]), but not the PDT onset temperature that is detected at $220 \pm 10 \mathrm{~K}$ for all samples (see the blue area in Fig. 1).

\subsection{DSC}

DSC upscans relative to myoglobin powders at various h values are reported in Fig. 2a. Note that a straight line obtained by fitting the data points in the 130-170 K temperature interval has been subtracted from the original, baseline subtracted, thermograms in order to highlight the relevant specific heat variations. As can be seen, a glass 


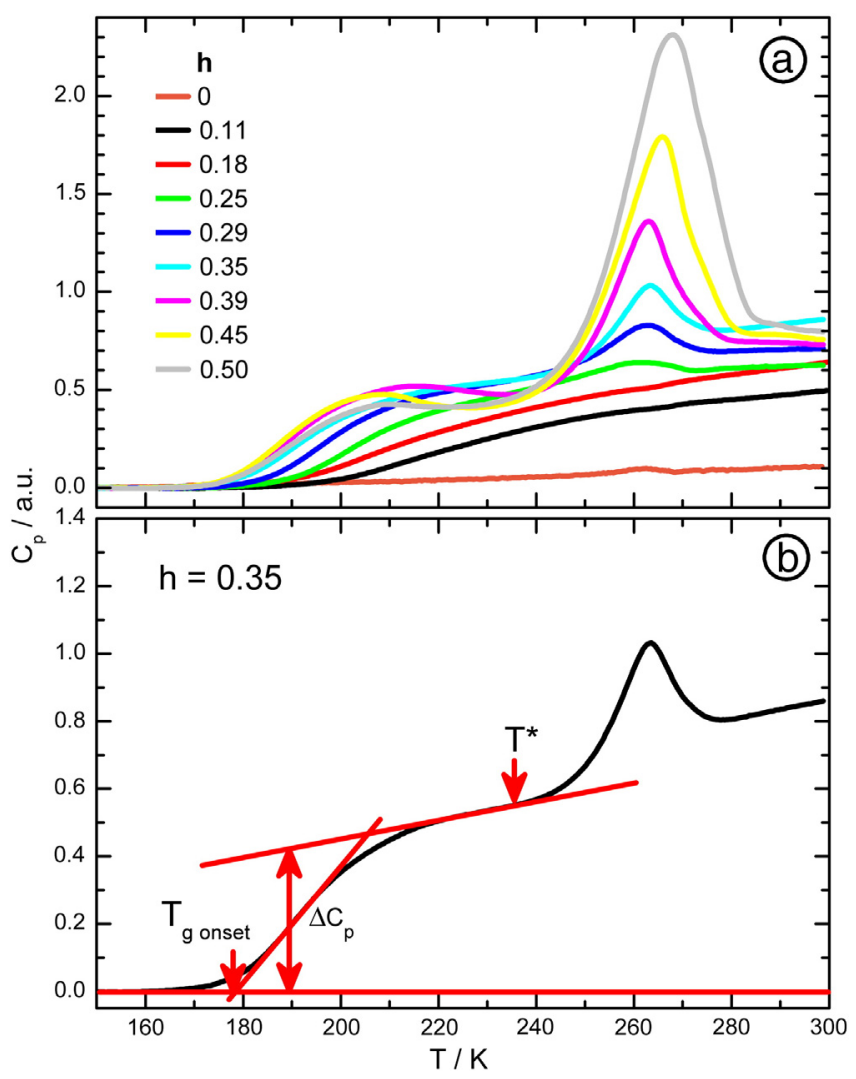

Fig. 2. Panel a: calorimetric upscans (scan rate $20 \mathrm{~K} / \mathrm{min}$ ) of Mb powders at selected representative hydrations. Panel b: pictorial representation of the procedure used to determine $\mathrm{T}_{\mathrm{g} \text { onset, }} \mathrm{T}^{*}$ and $\Delta \mathrm{C}_{\mathrm{P}}$.

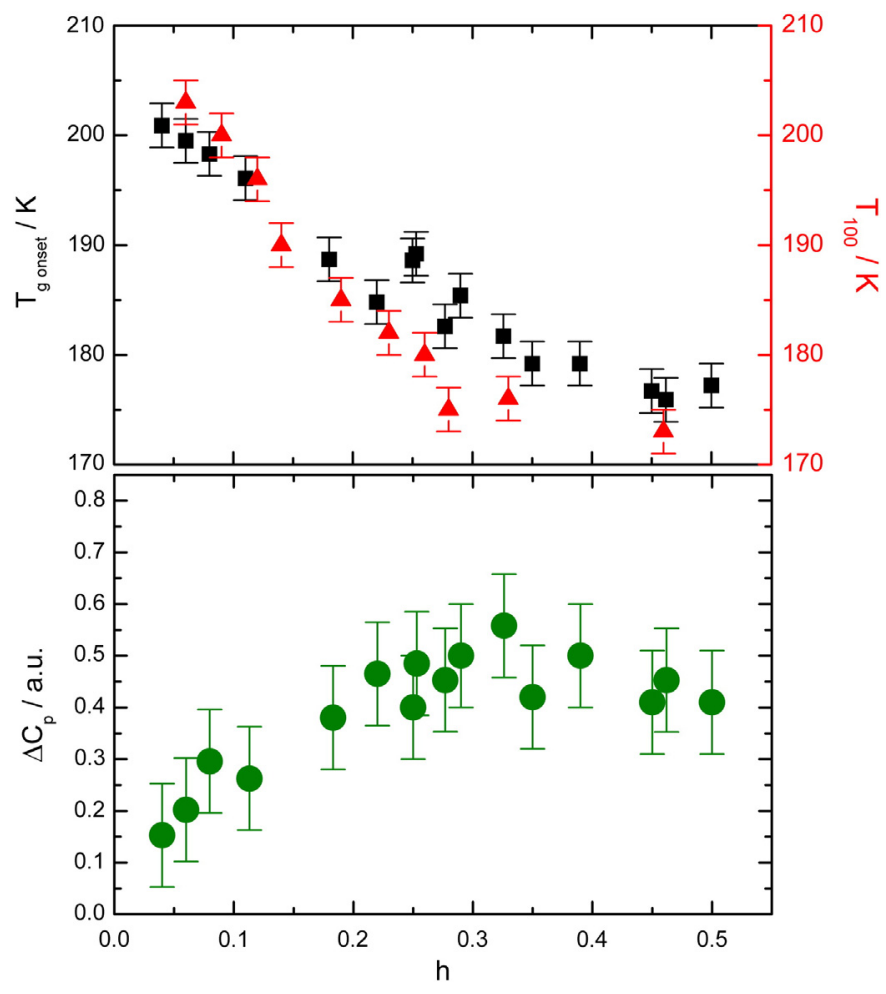

Fig. 3. Upper panel: $\mathrm{T}_{\mathrm{g} \text { onset }}$ (black squares, left scale) and $\mathrm{T}_{100}$ (red triangles, right scale) as a function of hydration. Lower panel: $\Delta C_{P}$ as a function of hydration. (For interpretation of the references to color in this figure legend, the reader is referred to the web version of this article.) transition is identified as a specific heat step in the lower temperature region of the thermograms; its onset temperature decreases with increasing hydration while the specific heat leap increases with hydration; both effects tend to reach a plateau at $h>0.30$. Panel $b$ illustrates the procedure used to determine the glass transition onset temperature ( $\left.T_{g \text { onset }}\right)$ and the specific heat leap $\left(\Delta C_{P}\right)$.

The absence of any transition in the dry $(h=0)$ sample (see panel a) and the fact that $\Delta C_{P}$ increases almost linearly with hydration in the range $0<\mathrm{h}<0.3$ (see also the lower panel of Fig. 3) suggests that the features observed in the hydrated samples refer to hydration water. The hydration dependence of the glass transition related quantities $T_{g}$ onset and $\Delta C_{P}$ is reported in Fig. 3, upper and lower panel, respectively.

Interestingly, a broad endothermic peak is observed for samples with $\mathrm{h}>0.2$. The onset of this peak is independent on hydration and is located at about $230 \div 240 \mathrm{~K}$, that we identify as $\mathrm{T}^{*}$, much lower that the melting temperature of bulk $\mathrm{D}_{2} \mathrm{O}(\sim 277 \mathrm{~K})$; at the highest hydrations investigated $(h>0.35)$ the peak broadens significantly towards the high temperatures and likely contains contributions from the melting of heavy water, in agreement with the suggestion that water in excess of the first hydration shell may freeze [29].

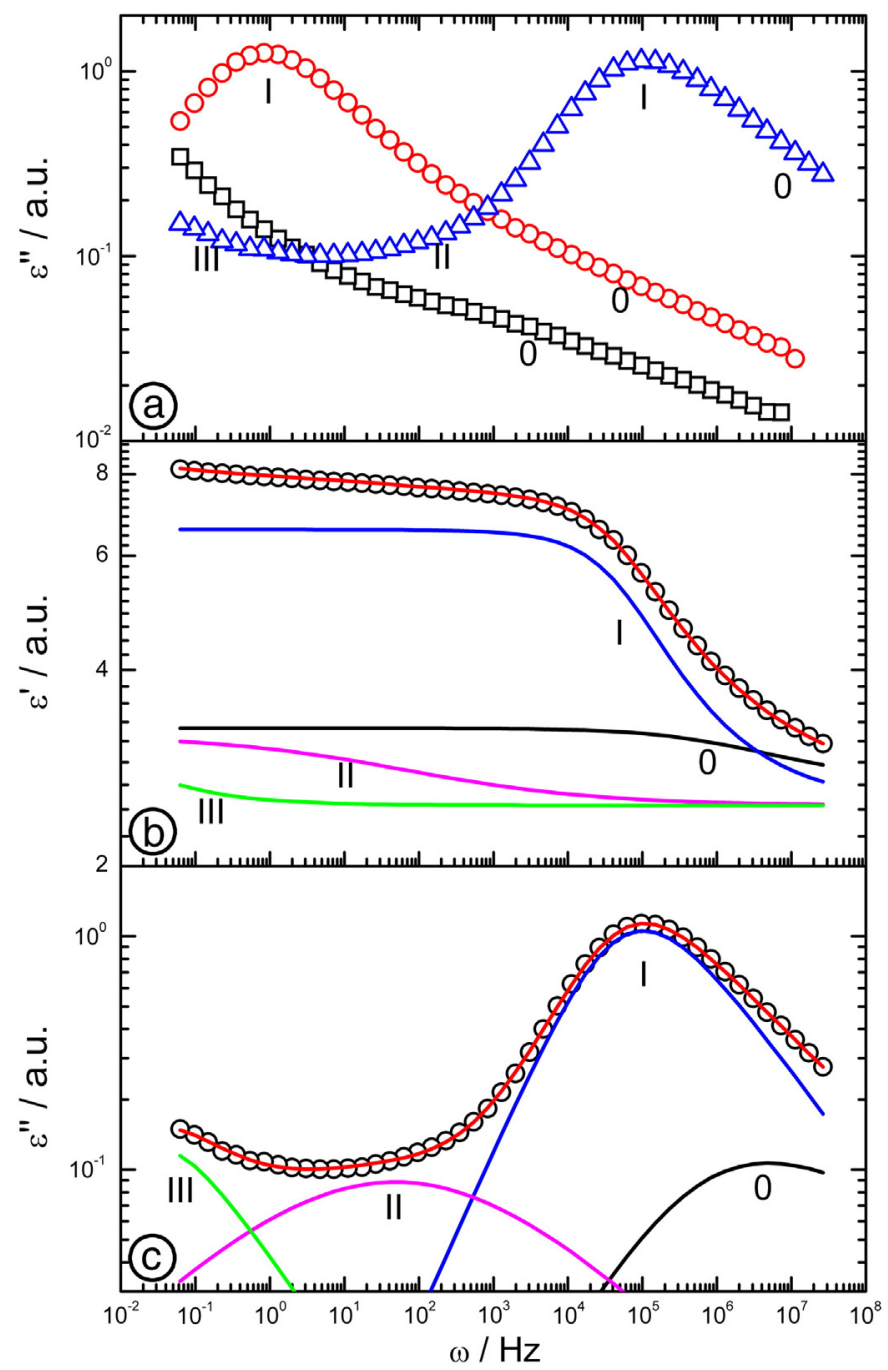

Fig. 4. Panel (a): dielectric losses in myoglobin powder at $\mathrm{h}=0.23$ and at $\mathrm{T}=173 \mathrm{~K}$ (black squares), $\mathrm{T}=188 \mathrm{~K}$ (red circles), $\mathrm{T}=218 \mathrm{~K}$ (blue triangles). Panel (b, c): Typical fit of the dielectric spectrum of $\mathrm{Mb}$ at $\mathrm{h}=0.23$ and $\mathrm{T}=218 \mathrm{~K}$ in terms of $\mathrm{HN}$ functions Upper: real part; lower: imaginary part. Continuous lines are overall fittings while dashed lines represent the individual processes. (For interpretation of the references to color in this figure legend, the reader is referred to the web version of this article.) 
Data in Fig. 3 clearly show that the glass transition onset temperature decreases with hydration while the specific heat leap increases; however, both effects tend saturate for hydration levels higher than about 0.3 . Considering that, for myoglobin, $h=0.3$ corresponds to full coverage of the first hydration shell [30], this confirms that the glass transition observed with calorimetry has to be related the protein hydration water (although involvement of protein degrees of freedom, as suggested in recent literature [14,31], cannot be excluded).

\subsection{BDS}

Dielectric loss spectra of myoglobin powders at $\mathrm{h}=0.23$ and at three representative temperatures are reported in Fig. 4a. At low temperatures a single weak and asymmetric relaxation is observed, that we call process " 0 ". This process is very similar to that observed by other groups for lysozyme ("fast" or "main" process [13,32]; process "1"[25]); it was not detected in a previous study by our group [17] due to lower sensitivity. As the temperature is increased a second, main, relaxation that we call process "I" steps in, followed by a third (process "II") relaxation; further lower frequency relaxations (like e.g. process "III") that enter in our frequency window at higher temperatures are neglected in the present work, in view of the uncertainties introduced by teflon coating (see below). In Fig. 4b and c we report a fit of the dielectric spectrum (both real and imaginary parts) in terms HN functions, as described in the Materials and Methods section. The analysis is fully compatible with that reported in ref. 17. Fittings of

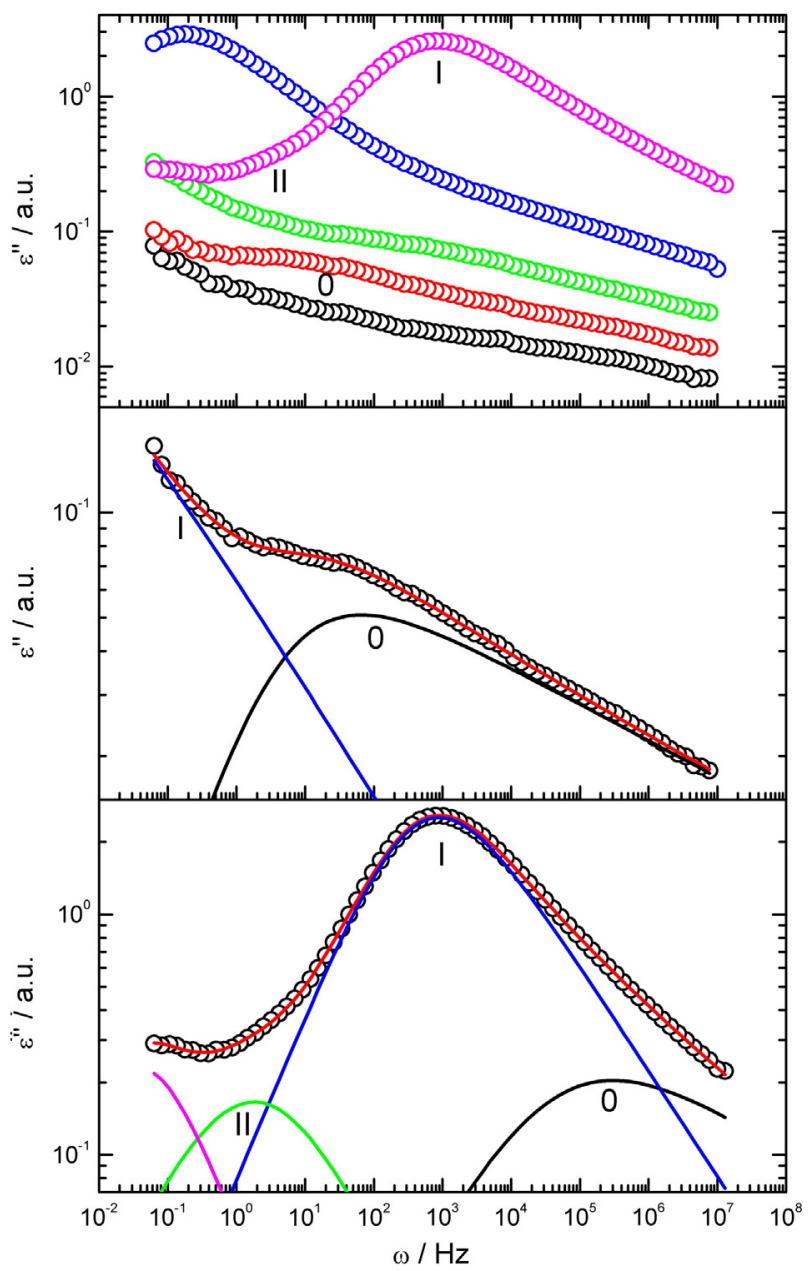

comparable quality are obtained at all hydrations and temperatures. As reported in the Materials and Methods section, we used teflon coated cells; use of teflon coating has been questioned in the literature, as it may introduce spurious peaks, especially at low frequencies and high temperatures $[22,33]$ and references therein. Therefore, to check the reliability of our results, we performed an "ad hoc" experiment whereby the same sample at $\mathrm{h}=0.2$ was measured in a teflon coated and in a gold coated cell. Results are reported in Fig. 5 and show that while with the teflon coated cell the relaxation peaks are clearly identified, with the gold coated cell they are almost completely obscured by a huge conductivity signal (except process 0 at low temperatures); however, the fittings reported in the middle and bottom panels indicate that no alterations of peak positions are introduced by teflon coating.

The temperature dependence of relaxation times for processes 0 , I and II is reported in Fig. 6 in the usual $\log \tau$ vs. 1000/T representation. Note that, when discussing dielectric data, one should keep in mind that all relaxing dipoles in the sample contribute to the observed signal; in the case of protein hydrated powders, both hydration water, polar protein side chains and backbone dipoles may contribute. Moreover, in view of the tight protein-hydration water coupling in our samples, separation of water and protein contributions may be often questionable.

Keeping in mind the above warnings, in agreement with previous studies $[13,25]$ we attribute process 0 as mainly arising from the fast rotational relaxation of $\mathrm{D}_{2} \mathrm{O}$ molecules in the first hydration shell; this is confirmed by the fact that its amplitude increases with increasing

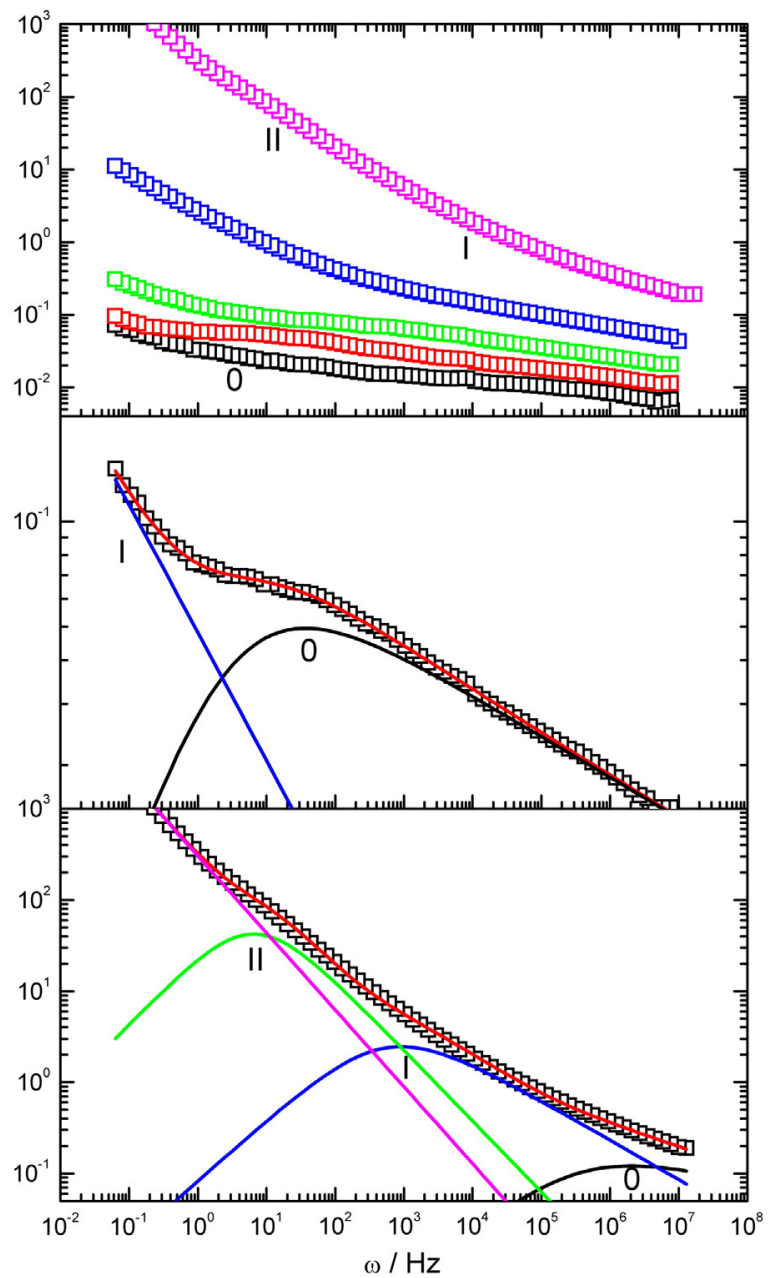

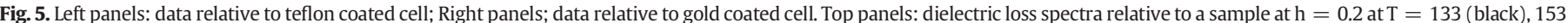

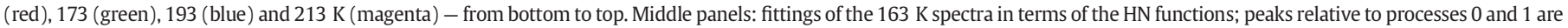

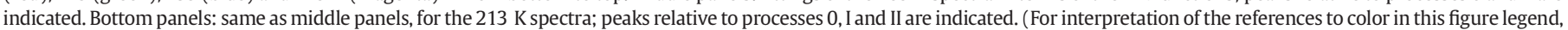
the reader is referred to the web version of this article.) 

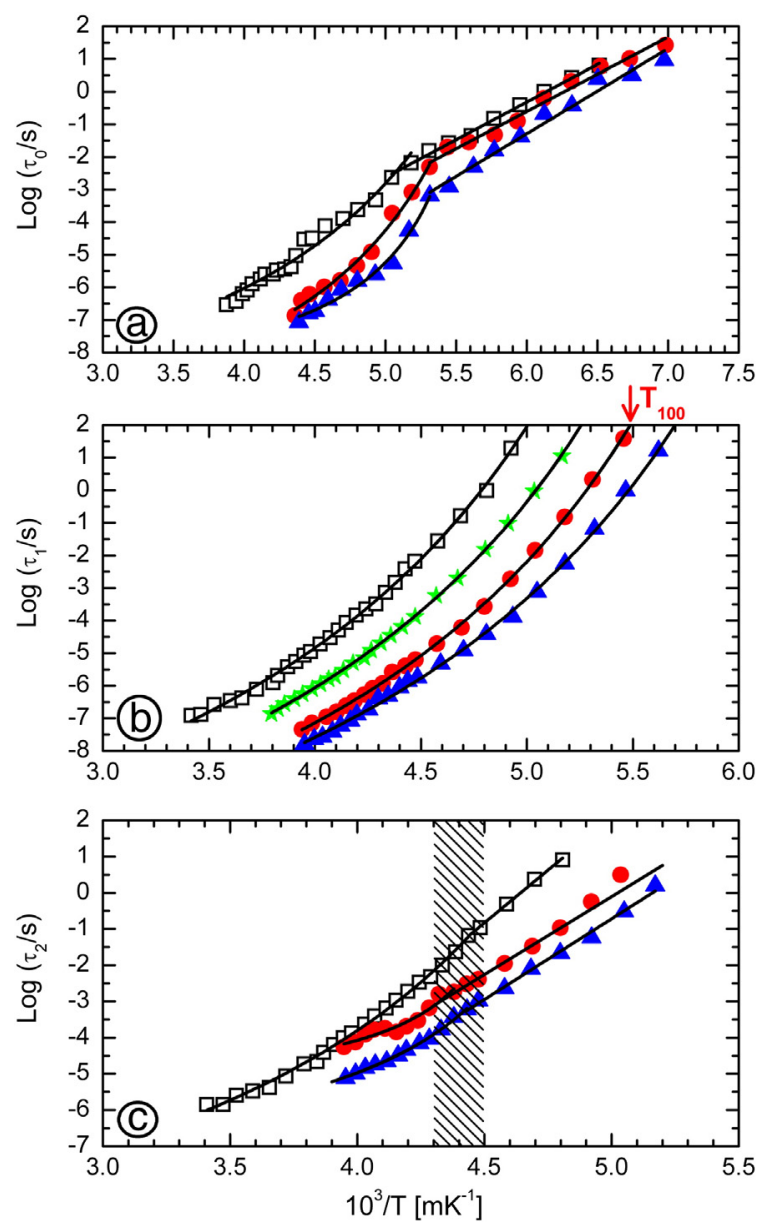

Fig. 6. Logarithm of $\tau_{0}$ (panel a), $\tau_{1}$ (panel b) and $\tau_{2}$ (panel c) as a function of the inverse temperature. Black open squares: $h=0.09$; green stars: $h=0.14$; red circles: $h=0.23$; blue triangles: $h=0.33$. The continuous lines are fittings in terms of the VFT or Arrhenius laws. The dashed zone in panel c highlights the temperature interval in which, for process II, a VFT-Arrhenius crossover is observed. (For interpretation of the references to color in this figure legend, the reader is referred to the web version of this article.)

hydration (data not shown). The temperature dependence of the relaxation time of process 0 , for lysozyme, has been analyzed as a simple $\mathrm{Ar}$ rhenius behavior with activation energy of $\sim 60 \mathrm{~kJ} / \mathrm{mol}[3,13]$ or as a more complex behavior, exhibiting a Vogel-Fulcher-Tamman (VFT)Arrhenius crossover at $180 \mathrm{~K}$ [25]. Our data (see panel a of Fig. 6) are compatible with this last behavior; we note, however, that process 0 is very weak and that at high temperatures $(>200 \mathrm{~K})$ it is partially obscured by the much larger process I: this may cause uncertainties in the exact determination of its temperature dependence at high T. In any case, a clear hydration dependence is observed and the slope of the Arrhenius behavior at low $\mathrm{T}$ gives an activation energy value of $\sim 50 \mathrm{~kJ} / \mathrm{mol}$. The hydration dependence observed for process 0 is analogous to that observed in ref. [13] for the "main" relaxation of hydrated lysozyme powders; in view of the ENS data reported in Fig. 1, this fact does not support the suggestion in ref. [3] that the PDT is related to the characteristic time of this process entering the time window of the neutron spectrometer used.

The temperature dependence of the relaxation time of process I is reported in Fig. 6b; at all hydrations it exhibits a VFT behavior, implying that we are observing a collective behavior of the protein + hydration water system. A collective process exhibiting a similar VFT behavior has also been observed for water confined in a disordered silica matrix [34]. For this process a marked hydration effect is observed: the curves shift to lower temperatures (higher $10^{3} / \mathrm{T}$ values) as h increases. From the data, the values of $\mathrm{T}_{100}$ (i.e. the temperature values at which the relaxation time of process I reaches the value of $100 \mathrm{~s}$ (which is the standard definition of the "dielectric" glass transition) can be obtained; $\mathrm{T}_{100}$ values as a function of hydration are reported in Fig. 3 panel a, in comparison with the calorimetric glass transition onset temperatures. The close similarity between the two sets of data suggests that process I is in fact a collective relaxation of the system, involved in the glass transition.

Concerning process II, we note that its hydration dependence is weaker than for process I; moreover, at all the hydrations investigated, it exhibits a VFT-Arrhenius crossover in the range $220 \div 230 \mathrm{~K}$ (evidenced by the dashed region in Fig. 6c), i.e. in the same temperature region where the onset of the PDT is observed. Following refs. [17] and $[35,36]$ we attribute process II mainly to relaxations of protein side chains strongly coupled to the dynamics of hydration water.

\section{Conclusions}

In this paper we have investigated the hydration dependence of mean square displacements, calorimetric glass transition and dielectric relaxations in myoglobin hydrated powders. The main results can be summarized as follows:

1) The onset temperature of the so called protein dynamical transition does not show any hydration dependence and occurs in the range $220 \pm 10 \mathrm{~K}$ in the whole hydration range explored. At difference, the MSD amplitudes observed at high temperature increase with increasing hydration.

2) The calorimetric glass transition onset temperature decreases with increasing hydration, the effect being clearly evident in the range $0<\mathrm{h}<0.3$. This behavior argues against the direct dependence of the PDT onset temperature upon the glass transition of the hydration water + protein system.

3) Process 0 observed with BDS exhibits a dependence upon hydration, both in intensity and relaxation times; this last observation, in view of the hydration independence of the PDT onset temperature, does not support the suggestion that the PDT observed with ENS is a mere resolution effect linked to the entry of this relaxation within the time window of the neutron spectrometer used.

4) Concerning process I, it has a marked hydration dependence and speeds up as hydration is increased; its collective VFT behavior and the close correspondence between the dielectric $T_{100}$ values and the $T_{g}$ onset values determined by calorimetry suggest that this process reflects a cooperative relaxation of the protein + hydration water system, directly involved in the glass transition of the system.

5) Concerning process II, the temperature dependence of its relaxation time is characterized by a VFT-Arrhenius crossover occurring in the same temperature range as the PDT onset. Although an overall hydration dependence of process II is observed, the above crossover occurs in the $210 \div 230 \mathrm{~K}$ interval, irrespective of the hydration. Following the suggestion by Schirò et al., 2012, 2013 [8,9] we may speculate that this crossover could reflect a change in proteinhydration water dynamics related to the putative LDL $\rightarrow$ HDL structural transition that has been suggested to occur in the hydration water of proteins [7] and in water confined in a silica matrix [37] that, in turn, could be the physical effect underlying the onset of the so called protein dynamical transition $[8,9,18]$.

Overall, the effect of increasing hydration (at least in the range $0<\mathrm{h}<0.3$ ) is to speed up myoglobin-hydration water relaxations; this is mirrored by the fact that the amplitudes of anharmonic mean square fluctuations increase with hydration and, thermodynamically, by the decrease of glass transition onset temperatures. On the other hand, the onset temperature of the myoglobin PDT is almost hydration independent; calorimetrically this is connected with the hydration independence of $\mathrm{T}^{*}$ and, dielectrically, with the VFT-Arrhenius crossover observed for process II. It is therefore fascinating to suggest that the PDT, in myoglobin hydrated powders, may be the dynamical 
counterpart of a structural Liquid-Liquid Phase Transition occurring in the hydration water.

That the anharmonic dynamics and the large scale structural fluctuations (and therefore the stability) of proteins depend on the physical properties of hydration water may be a general concept of potential industrial or even medical/clinical interest. If generalized and extended to other proteins and/or to systems more similar to the "in vivo" ones (e.g. to confined/crowded systems like proteins embedded in water/ sugar matrices at various hydrations $[38,39])$ the present results may pave the way to new applications in the fields of storage and cryopreservation of biological molecules or even tissues.

\section{Acknowledgements}

This work was supported by MIUR (Grant PRIN 2008ZWHZJT "Struttura-Dinamica-Funzione di Biomolecole in Sistemi lontani dall'Idealità Termodinamica"). The authors acknowledge the Institut Laue-Langevin (Grenoble, France) for beamtime allocation at IN13 station and Francesca Natali (CNR, ILL, Grenoble) for scientific support during ENS experiments.

\section{References}

[1] H. Frauenfelder, F. Parak, R.D. Young, Conformational substates in proteins, Annu. Rev. Biophys. Biophys. Chem. 17 (1988) 451-479.

[2] W. Doster, S. Cusack, W. Petry, Dynamical transition of myoglobin revealed by inelastic neutron scattering, Nature 337 (1989) 754-756.

[3] S. Khodadadi, S. Pawlus, J.H. Roh, V. Garcia Sakai, E. Mamontov, A.P. Sokolov, The origin of the dynamic transition in proteins, J. Chem. Phys. 128 (2008) 195106.

[4] G. Chen, P.W. Fenimore, H. Frauenfelder, F. Mezei, J. Swenson, R.D. Young, Protein fluctuations explored by inelastic neutron scattering and dielectric relaxation spectroscopy, Philos. Mag. 88 (2008) 3877-3883.

[5] S. Magazù, F. Migliardo, A. Benedetto, Puzzle of protein dynamical transition, J. Phys. Chem. B 115 (2011) 7736-7743.

[6] G. Zaccai, How soft is a protein? A protein dynamics force constant measured by neutron scattering, Science 288 (2000) 1604-1607.

[7] S.-H. Chen, L. Liu, E. Fratini, P. Baglioni, A. Faraone, E. Mamontov, Observation of fragile-to-strong dynamic crossover in protein hydration water, Proc. Natl. Acad. Sci. 103 (2006) 9012-9016

[8] G. Schirò, F. Natali, A. Cupane, Physical origin of anharmonic dynamics in proteins: new insights from resolution-dependent neutron scattering on homomeric polypeptides, Phys. Rev. Lett. 109 (2012) 128102-128106

[9] G. Schirò, Anharmonic onsets in polypeptides revealed by neutron scattering: Experimental evidences and quantitative description of energy resolution dependence, Biophys. Chem. 180-181 (2013) 29-36.

[10] K.L. Ngai, S. Capaccioli, A. Paciaroni, Change of caged dynamics at $\mathrm{T}_{\mathrm{g}}$ in hydrated proteins: Trend of mean squared displacements after correcting for the methyl-group rotation contribution, J. Chem. Phys. 138 (2013) 235102-235115.

[11] W. Doster, The two-step scenario of the protein dynamical transition, J. Non-Cryst. Solids 357 (2011) 622-628.

[12] A.M. Klibanov, Improving enzymes by using them in organic solvents, Nature 409 (2001) 241-246.

[13] S. Khodadadi, S. Pawlus, A.P. Sokolov, Influence of hydration on protein dynamics: combining dielectric and neutron scattering spectroscopy data, J. Phys. Chem. B 112 (2008) 14273-14280.

[14] H. Jansson, R. Bergman, J. Swenson, Role of solvent for the dynamics and the glass transition of proteins, J. Phys. Chem. B 115 (2011) 4099-4109.
[15] J.A. Rupley, E. Gratton, G. Careri, Water and globular proteins, Trends Biochem. Sci. 8 (1983) 18-22.

[16] J.A. Rupley, G. Careri, Protein hydration and function, Adv. Protein Chem. 41 (1991) 37-172.

[17] G. Schirò, A. Cupane, E. Vitrano, F. Bruni, Dielectric relaxations in confined hydrated myoglobin, J. Phys. Chem. B 113 (2009) 9606-9613.

[18] G. Schirò, M. Fomina, A. Cupane, Communication: Protein dynamical transition vs. liquid-liquid phase transition in protein hydration water, J. Chem. Phys. 139 (2013) 121102.

[19] G. Schirò, M. Sclafani, C. Caronna, F. Natali, M. Plazanet, A. Cupane, Dynamics of myoglobin in confinement: An elastic and quasi-elastic neutron scattering study, Chem. Phys. 345 (2008) 259-266.

[20] G. Schirò, M. Sclafani, F. Natali, A. Cupane, Hydration dependent dynamics in sol-gel encapsulated myoglobin, Eur. Biophys. J. 37 (2008) 543-549.

[21] F. Gabel, D. Bicout, U. Lehnert, M. Tehei, M. Weik, G. Zaccai, Protein dynamics studied by neutron scattering, Q. Rev. Biophys. 35 (2002) 327-367.

[22] R. Richert, Insulated electrodes for eliminating conductivity in dielectric relaxation experiments, Eur. Phys. J. B 68 (2009) 197-200.

[23] H. Jansson, R. Bergman, J. Swenson, Hidden slow dynamics in water, Phys. Rev. Lett. 104 (2010) 017802.

[24] R. Richert, Comment on "Hidden Slow Dynamics in Water", Phys. Rev. Lett. 104 (2010) 249801

[25] F. Bruni, R. Mancinelli, M.A. Ricci, Multiple relaxation processes versus the fragile-to-strong transition in confined water, Phys. Chem. Chem. Phys. 13 (2011) 19773-19779.

[26] G. Höhne, W.F. Hemminger, H.-J. Flammersheim, Differential Scanning Calorimetry, second ed. Springer, Germany, 2003.

[27] G. Schirò, C. Caronna, F. Natali, A. Cupane, Molecular origin and hydration dependence of protein anharmonicity: an elastic neutron scattering study, Phys. Chem. Chem. Phys. 12 (2010) 10215-10220.

[28] A. Paciaroni, S. Cinelli, E. Cornicchi, A. De Francesco, G. Onori, Fast fluctuations in protein powders: The role of hydration, Chem. Phys. Lett. 410 (2005) 400-403.

[29] G. Sartor, A. Hallbrucker, E. Mayer, Characterizing the secondary hydration shell on hydrated myoglobin, hemoglobin, and lysozyme powders by its vitrification behavior on cooling and its calorimetric glass $\rightarrow$ liquid transition and crystallization behavior on reheating, Biophys. J. 69 (1995) 2679-2694.

[30] V. Lounnas, B.M. Pettitt, A connected-cluster of hydration around myoglobin: correlation between molecular dynamics simulations and experiment, Proteins 18 (1994) 133-147.

[31] H. Jansson, J. Swenson, The protein glass transition as measured by dielectric spectroscopy and differential scanning calorimetry, Biochim. Biophys. Acta 1804 (2010) 20-26.

[32] S. Khodadadi, J.E. Curtis, A.P. Sokolov, Nanosecond relaxation dynamics of hydrated proteins: water versus protein contributions, J. Phys. Chem. B 115 (2011) 6222-6226

[33] P.B. Ishai, M.S. Talary, A. Caduff, E. Levy, Y. Feldman, Electrode polarization in dielectric measurements: a review, Meas. Sci. Technol. 24 (2013) 102001-102021.

[34] M. Cammarata, M. Levantino, A. Cupane, A. Longo, A. Martorana, F. Bruni, Structure and dynamics of water confined in silica hydrogels: X-ray scattering and dielectric spectroscopy studies, Eur. Phys. J. E 12 (2003) S63-S66.

[35] H. Jansson, R. Bergman, J. Swenson, Relation between solvent and protein dynamics as studied by dielectric spectroscopy, J. Phys. Chem. B 109 (2005) 24134-24141.

[36] J. Swenson, H. Jansson, R. Bergman, Relaxation processes in supercooled confined water and implications for protein dynamics, Phys. Rev. Lett. 96 (2006) 247802-247805

[37] L. Liu, S.-H. Chen, A. Faraone, C.-W. Yen, C.-Y. Mou, Pressure dependence of fragile-to-strong transition and a possible second critical point in supercooled confined water, Phys. Rev. Lett. 95 (2005) 117802.

[38] G. Bellavia, S. Giuffrida, G. Cottone, A. Cupane, L. Cordone, Protein thermal denaturation and matrix glass transition in different protein-trehalose-water systems, J. Phys. Chem. B 115 (2011) 6340-6346.

[39] G. Bellavia, S. Giuffrida, G. Cottone, A. Cupane, L. Cordone, Thermal denaturation of myoglobin in water-disaccharide matrixes: relation with the glass transition of the system, J. Phys. Chem. B 113 (2009) 11543-11549. 